\title{
Protocolo de muestreo para la estimación de la diversidad de murciélagos con redes de niebla en estudios de ecología
}

\author{
JuLio C. BRACAMONTE \\ Centro de Investigaciones Básicas y Aplicadas, Facultad de Ingeniería, Universidad Nacional de Jujuy. San Salvador de Jujuy, \\ Jujuy, Argentina.
}

\begin{abstract}
RESUMEN. Los murciélagos constituyen una proporción considerable de la mastofauna del Neotrópico, por lo que estudiarlos resulta crucial para comprender los procesos ecológicos que tienen lugar en sus ecosistemas. Para tal fin, el método de captura de murciélagos con redes de niebla es el más usado y reconocido. Sin embargo, quienes quieren emplear esta técnica suelen desconocer los pormenores de su uso; además, los grupos de investigación no siempre incluyen personas experimentadas para llevar adelante las capturas y para transmitir sus conocimientos en la materia. En este trabajo pongo a disposición de estudiantes e investigadores noveles en el uso de redes de niebla un protocolo que facilitará su desempeño y permitirá obtener resultados comparables con otros estudios. Por otra parte, resalto la necesidad de considerar el bienestar de los animales objeto de estudio al momento de hacer capturas.
\end{abstract}

[Palabras clave: Chiroptera, método de captura, diversidad, comunidad]

\begin{abstract}
AвstRACt. Sampling protocol for the estimation of bat diversity with mist nets in ecological studies. Bats represent a considerable proportion of the mammals of the Neotropics; thus, it is important to understand the ecological processes that occur in their ecosystems. For such purposes, the capture of bats with the mist-netting method has been more intensively used and is widely recognized. However, research groups do not always include experienced people to carry out the trapping and to transmit their knowledge on the subject. In this work, I make available both for students and researchers who are new to the use of mist-netting a protocol that will facilitate their performance and will allow obtaining results comparable with other studies. On the other hand, I highlight the need to consider the welfare of the animals under study at the time of capturing.
\end{abstract}

[Keywords: Chiroptera, capture method, diversity, community]

\section{INTRODUCCIÓN}

Los murciélagos constituyen el orden de mamíferos ecológicamente más diverso del planeta con 1223 especies distribuidas casi globalmente (Schipper etal.2008). Representan una alta proporción de los ensambles de mamíferos del Neotrópico (Simmons and Voss 1998). Ocupan una amplia diversidad de nichos tróficos que los posiciona en diferentes niveles de las cadenas alimentarias y en el seno de procesos ecológicos fundamentales como la polinización, la dispersión de semillas y la regulación de poblaciones de insectos (Fleming et al. 2009; Kunz et al. 2011). Además, su dependencia de ambientes naturales los hace muy sensibles a la pérdida de hábitat, que genera cambios en la composición de especies (Medellín et al. 2000; Estrada and Coates-Estrada 2002; Peters et al. 2006) y que los convierte en potenciales indicadores de la calidad del ambiente (Jones et al. 2009).
A pesar de tener una distribución cosmopolita, la mayor diversidad de murciélagos se concentra en los trópicos y disminuye conforme aumenta la latitud (Patterson et al. 2003). Sin embargo, a pesar de ser menor la diversidad de murciélagos puede ser difícil de estimar, incluso en latitudes más altas, por características propias del grupo como hábitos nocturnos, capacidad de vuelo y pequeño tamaño. No obstante, generar información sobre el elenco faunístico de diferentes áreas es primordial para establecer líneas de base y planes de manejo o monitoreos de fauna. Esta información debería ser incluida en evaluaciones de impacto ambiental.

Los subtrópicos, entre $30^{\circ}$ y $35^{\circ} \mathrm{S}$, representan los límites australes para la distribución de numerosas especies y familias de murciélagos (McNab 1982). En torno a estas latitudes se encuentra la Argentina, que contiene, hasta 
el momento, 62 especies de murciélagos (Barquez et al. 2006; Barquez et al. 2009; Díaz and Barquez 2009). Esta ubicación determina bajas riqueza y abundancias relativas $\mathrm{y}$, por lo tanto, la existencia de ensambles particulares de murciélagos (Barquez and Díaz 2001). Al mismo tiempo, la estacionalidad podría resultar un factor importante en la composición de ensambles de murciélagos (Klingbeil and Willig 2010). En consecuencia, la metodología de captura debería ajustarse a las circunstancias y diversidad de murciélagos existente en latitudes como las de la Argentina.

El estudio de murciélagos está tomando un impulso notable en los últimos años en la Argentina (Barquez et al. 2009; Díaz and Barquez 2009; Bracamonte 2010, 2013; Sandoval et al. 2010; Sánchez et al. 2012a,b; Sánchez and Giannini 2013; Bracamonte and Lutz 2013; López Berrizbeitia and Díaz 2013; Sandoval and Barquez 2013; Castilla et al. 2013), donde, al igual que en otras partes del mundo, la captura con redes de niebla es el método más empleado a la hora de realizar estudios de diversidad, a pesar de los sesgos y limitaciones que se reconocen (Kunz et al. 2009). Resulta útil sugerir la implementación de un procedimiento estándar para quienes deseen iniciarse en la temática. Por ello, el objetivo de este trabajo es proponer un protocolo de muestreos con redes de niebla que ayude a estudiantes e investigadores jóvenes a iniciar estudios ecológicos en murciélagos. De esta manera se pretende proponer pautas generales basadas en experiencia empírica y en la revisión de la información de fondo disponible en cuanto al empleo de las redes de niebla. Este protocolo permitirá el desarrollo y ejecución de diseños experimentales que permitan obtener datos comparables entre estudios, además de considerar el bienestar de los animales.

Las redes de niebla, originalmente utilizadas para la captura de aves, son el elemento más empleado en la actualidad para capturar murciélagos. Suelen estar confeccionadas con una malla de nylon sostenida por hilos tensores horizontales de mayor grosor, que forman "bolsillos" en su parte inferior y que son el lugar donde caen los individuos que no quedan enredados en la malla. Los tensores, por lo general cuatro, poseen anillas o soportes en cada extremo que sirven para sostener a la red de los elementos que cumplen la función de sostén (Figura 1). El tamaño de la malla es variable, aunque las medidas más empleadas están en el rango de 30 a $36 \mathrm{~mm}$, lo que ha dado buenos resultados en las capturas en la Argentina. La longitud de las redes también es variable, pero las más comúnmente empleadas son las de 6, 9 y $12 \mathrm{~m}$ de largo x 2.5 ó $3 \mathrm{~m}$ de alto. Existen redes de mayor longitud y altura, más difíciles de manejar y de colocar (Rinehart and Kunz 2001).

\section{VENTAJAS, DESVENTAJAS Y SESGOS}

El uso de redes permite capturar individuos para confirmar la identidad sistemática de las especies, ya que se dispone de ejemplares en la mano, además de recabar datos adicionales en cuanto a su morfometría, dieta o reproducción. Entre las ventajas operativas de las redes de niebla se destaca que son muy livianas y comprimibles, por lo que son fáciles de transportar al no requerir ni gran espacio ni mucha logística. Al mismo tiempo, esto permite llevarlas a sitios de difícil acceso, a diferencia de otros implementos con estructuras de metal, complicados para transportar. Además, al ser sencillas para instalar, se las puede adaptar a casi todo tipo de hábitat; incluso, se pueden ensamblar varias redes en serie para abarcar áreas más grandes (Kunz et al. 2009). Sin embargo, si el número de redes es elevado, el tiempo de colocación también será considerable. Su manejo, y en particular la extracción de los animales atrapados, requiere destreza y experiencia por parte de la persona que las utiliza. Las redes de niebla no suelen tener una vida útil prolongada cuando se emplean para la captura de murciélagos, ya que estos animales pueden masticarlas y destruirlas en poco tiempo, en el intento de liberarse. Las redes de niebla son más eficaces para la captura de murciélagos frugívoros (familia Phyllostomidae), ya que las especies insectívoras, por ejemplo, integrantes de las familias Vespertilionidae y Molossidae, son menos propensas a ser capturadas con este método (Simmons and Voss 1998). En la Argentina, las especies de estas familias son dominantes, en particular al centro y sur del país (Barquez et al. 1999; Castilla et al. 2013), por lo que, además, en estas zonas resulta altamente eficiente complementar el uso de redes con otros métodos de captura (e.g., trampas harpa, redes de mariposa, redes en las salidas o en las cercanías de refugios en viviendas). A pesar de todo esto, la eficiencia de las redes depende mucho de la experiencia del investigador y de su conocimiento sobre los hábitats frecuentados, hábitos y horarios de actividad de las especies; todo esto es de 


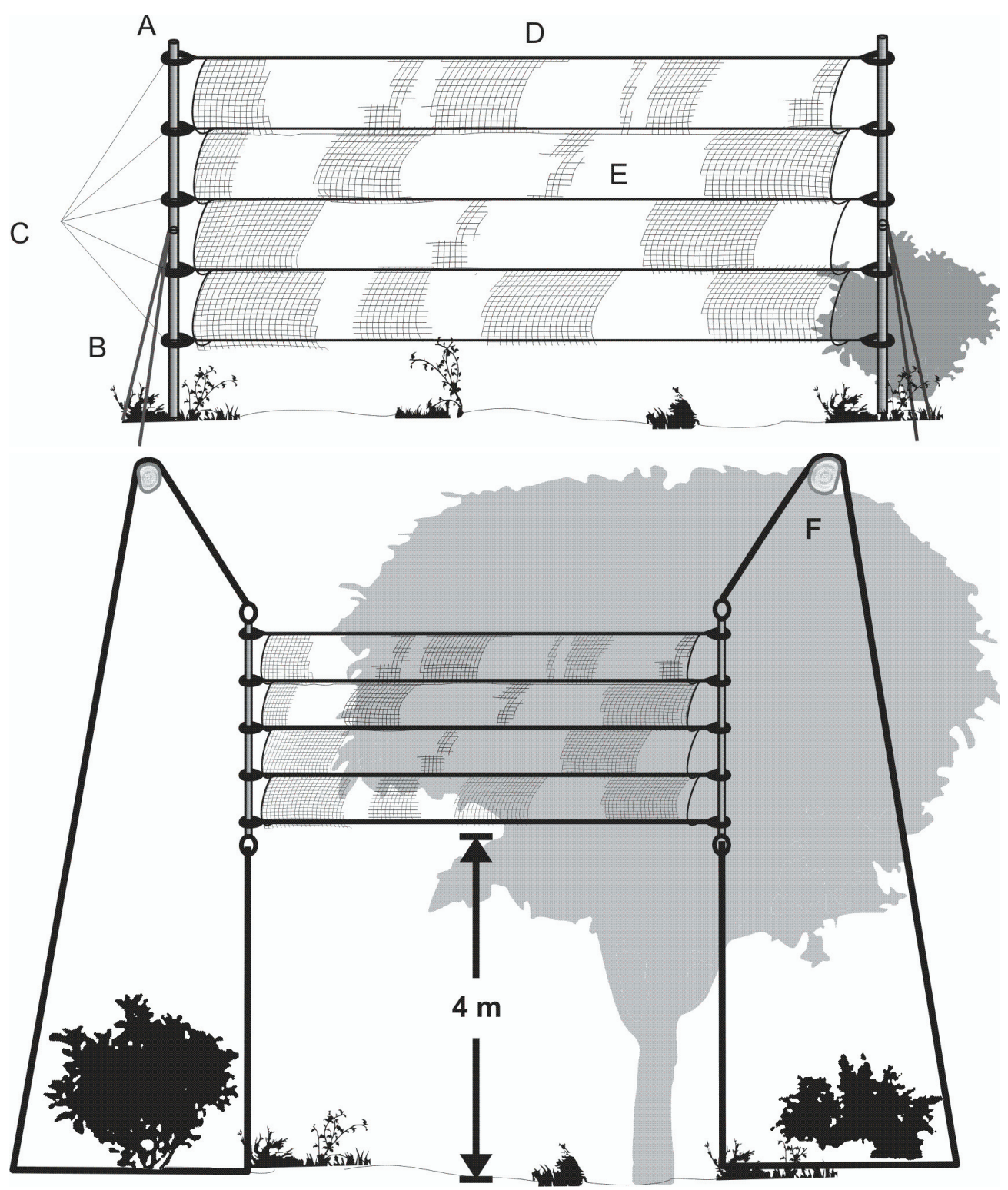

Figura 1. Dos modos de colocar una red de niebla. 1) Al nivel del suelo. 2) Elevada al nivel de sotobosque o dosel. A) Sostenes o parantes de la red; B) tensores de los parantes atados a estacas; C) anillos de la red; D) tensor horizontal de la red que define los cuatro paños; E) malla; F) tronco o rama de árbol. Obsérvese la presencia de "bolsillos" o pliegues en la parte inferior de cada paño.

Figure 1. Two ways to place a mistnet. 1) At the ground level. 2) Elevated at the understory or canopy level. A) Steel poles or shelf of the mistnet; B) ropes attached to poles and tied to stakes; C) rings of the mistnet; D) horizontal line of the mistnet that defines four cloths; E) mesh; F) trunk or tree branch. Note the presence of "pockets" or folds at the bottom of each cloth.

gran ayuda a la hora de buscar los mejores sitios para colocarlas.

\section{COLOCACIÓN}

El objetivo del estudio (diversidad, muestreos poblacionales, dieta, ectoparásitos, etc.) tiene relación con el lugar donde se colocarán las redes. Esto puede ser a nivel del suelo o elevadas usando un sistema de poleas que permite elevar las redes a diferentes alturas (Tschapka 1998; Rinehart and Kunz 2001; Kunz and Kurta 1988; Kunz et al. 2009) (Figura 1). En el mercado existen, además, soportes telescópicos para elevar las redes a diferentes alturas sin necesidad de usar árboles. Algunos autores aconsejan desplegar 10 redes de la misma o de diferentes medidas en una superficie de $500 \mathrm{~m}^{2}$ de apariencia homogénea (Galarza and Aguirre 2006) o en 
transectas lineales cada 40-50 m (Sánchez and Giannini 2013). Se recomienda usar redes de diferentes medidas $(6,9$ y $12 \mathrm{~m})$ para ajustarlas a los diferentes espacios que se pueden encontrar en un determinado sitio. Las redes se deben ubicar de tal manera de abarcar todos los microambientes posibles, como el interior y el borde de bosques o de los lugares que habitualmente emplean los murciélagos para desplazarse y donde, por lo tanto, hay más oportunidad de capturar especies (e.g., cuerpos de agua, arroyos, sendas, caminos y claros) (Palmeirim and Etheridge 1985; Kingston 2009; Frencke et al. 1987; Lisón and Calvo 2011).

Las redes son delicadas y necesitan de cuidado, por lo que previo a la colocación se debe limpiar la zona de ramas y arbustos que puedan dañarlas. Es preferible colocar las estacas y parantes primero, sostener un extremo de la red en uno de los parantes y extenderla hasta el otro para ajustar los tensores. En ese punto se puede aprovechar para realizar una limpieza adicional a fin de evitar enredos con plantas. Finalmente, se sigue el hilo principal (a menudo, de doble hilo) para que la red no quede cruzada y se la extiende para dar un ajuste final a los tensores de los parantes. La extensión de los paños no debe ser excesiva para que se formen los bolsillos en cada paño (Figura 1).

\section{REMOCIÓN DE LOS INDIVIDUOS CAPTURADOS}

Se debe remover con mucho cuidado los individuos capturados en las redes a fin de evitar dañarlos. Es importante usar guantes de cuero, dado que los murciélagos son potenciales vectores de la rabia (Constantine 2009). El investigador podrá usar guante sólo en la mano que sujetará el cuerpo del murciélago, de modo que la mano sin guante tenga más sensibilidad para desenredar los hilos. También se recomienda evitar el uso de ropa con botones, relojes u otros accesorios que puedan enredarse en la red y dificultar la extracción del animal. Antes de remover a un murciélago se recomienda definir el lado de la red por el cual ingresó (lo que se logra estirando la red por ambos lados) y ejecutar la remoción por ese mismo lado. Primero se desenredan las patas y la cola, luego el cuerpo y, por último, la cabeza y las alas (Finnemore and Richardson 2004).

\section{CONSIDERACIONES METODOLÓGICAS}

Cuando se usen redes para capturar murciélagos se deben tener en cuenta algunas consideraciones, a efectos de que los datos obtenidos se puedan comparar razonablemente con otros estudios. Para representar mejor las especies que frecuentan un tipo de ambiente se deben colocar varias redes en ese ambiente. La lluvia afecta de forma negativa la actividad de los murciélagos (Voigt et al. 2011), por lo que se deben evitar los muestreos bajo esas condiciones. Además, la lluvia, al mojar las redes, forma gotas que hacen que las redes sean más detectables por los murciélagos (Carvalho et al. 2011). Vientos fuertes y descensos bruscos de temperatura pueden alterar los niveles de actividad de estos animales (Jantzen and Fenton 2013). Las noches de luna llena pueden generar sesgos en las capturas por el fenómeno conocido como "fobia lunar" (Morrison 1978; Gannon and Willig 1997; Saldaña-Vázquez and MunguíaRosas 2013), que disminuye la actividad y restringe los movimientos de los murciélagos debido a que la claridad de la luna llena los hace más vulnerables a la depredación (Lang et al. 2006).

La actividad de los murciélagos no se distribuye uniformemente a lo largo de la noche. Es más alta poco después de la puesta del sol y durante la primera hora de la noche, de acuerdo con la dispersión de los animales desde sus refugios y el primer período de alimentación de las especies insectívoras (Anthony and Kunz 1977; Swift 1980; Rydell 1993). A menudo pueden ocurrir picos de actividad alrededor de la medianoche y hacia el amanecer (Autino and Barquez 1993; Rydell 1993). Por ello, la mayoría de las redes deben estar abiertas al comenzar la actividad de los murciélagos y permanecer así por seis horas, aprovechando el período de mayor actividad (Emmons et al. 2006), por toda la noche si las tasas de capturas son bajas (Bracamonte 2010) o por el tiempo estipulado para cumplir con los objetivos particulares del estudio planteado. Muestrear por toda la noche no siempre incrementa significativamente las capturas. Si existe la posibilidad de realizar muestreos preliminares para evaluar el diseño experimental, es conveniente mantener las redes funcionando toda la noche para una mejor comprensión de los niveles de actividad local de las especies (Esberard and Bergallo 2005) y para registrar especies raras, ya sea 
por poseer densidades poblacionales bajas o por sesgos de la metodología o especies que usualmente se incorporan de forma aleatoria durante el muestreo (Esberard 2009). Se requieren al menos de tres noches de muestreo en una localidad bajo buenas condiciones climáticas para tener una representación adecuada de la diversidad de especies (ver, sin embargo, consideraciones más abajo) (Galarza and Aguirre 2006). Se debe revisar las redes de forma periódica, con frecuencias a determinar (e.g., 20, 30 ó 60 minutos) según la abundancia y la diversidad detectada. Tiempos prolongados entre las revisiones pueden conducir al escape de individuos o, en el peor de los casos, a la muerte por hipotermia, estrés o depredación; además, aumenta la probabilidad de que las redes resulten dañadas (Bracamonte, observación personal).

Otra consideración cuando se realizan muestreos por varias noches consecutivas es el cambio diario de posición de cada red, ya que puede darse una disminución notable de las tasas de captura conforme pasan las noches si éstas se dejan en el mismo lugar (Thomas and LaVal 1988; Jouard 2007; Mello et al. 2008). Esto ocurre por aprendizaje de su ubicación y por evasión del área de muestreo (LaVal and Fitch 1977). El cambio de posición puede aumentar las probabilidades de registrar un mayor número de especies (Bergallo et al. 2003).

También debe tenerse en cuenta la estacionalidad en el área de muestreo al momento de diseñar el estudio. La estacionalidad puede manifestarse de diferentes maneras, como un cambio en la cantidad de precipitaciones, la temperatura y la duración del día; todo esto afecta la actividad de los murciélagos. Si bien los muestreos no deberían comenzar con temperaturas ambientales bajas, ante una imprevista baja de la temperatura (a menos de $10^{\circ} \mathrm{C}$ ) en épocas invernales o zonas elevadas, se recomienda cesar los muestreos porque en esas condiciones aumenta el riesgo de que los individuos capturados sufran hipotermia (Bracamonte, observación personal).

Por último, a efectos de hacer comparables nuestros resultados con los de otras investigaciones hay dos puntos clave a considerar. El primero es que los resultados del esfuerzo de muestreo y la tasa de captura sean expresados de forma correcta. El esfuerzo se cuantifica comúnmente como horas red, con un número determinado de redes (Fenton et al. 1992), o el de metros red por hora ( $\mathrm{mx}$ h) (Medellín 1993). Moreno y Halffter (2000) propusieron una modificación del método de Medellín; ellos calculan el esfuerzo como la suma de los metros de red por hora (m de $\mathrm{red} / \mathrm{h}$ ) para cada sitio de muestreo. La tasa de captura puede calcularse como el número de individuos capturados por cada $100 \mathrm{~h} / \mathrm{red}$ o cada $500 \mathrm{~m} / \mathrm{h}$, según corresponda. El segundo asunto importante es evaluar el grado de confianza o el nivel de representatividad que obtuvo el inventario realizado. Para evaluar esto analíticamente se pueden emplear curvas de acumulación de especies (Colwell and Coddington 1994). Visualmente, el alcance de valores asintóticos en las curvas de acumulación determina una mejor representatividad del conjunto de especies en una localidad (Longino et al. 2002). Para complementar este análisis con estimadores de riqueza no paramétricos se puede calcular la eficiencia del inventario como el porcentaje de especies observadas respecto a las determinadas por el estimador empleado. No es objeto de este trabajo entrar en detalles sobre estos métodos. Sin embargo, la literatura en este sentido es muy amplia y accesible para consultar (e.g., Magurran 2004 o Moreno 2001).

\section{Protocolo de CAPTURA CON REDES DE NIEBLA PARA LA ESTIMACIÓN DE LA DIVERSIDAD DE MURCIÉLAGOS EN UNA LOCALIDAD}

El siguiente es un protocolo para aplicar en ambientes boscosos subtropicales con el objetivo de lograr una mejor estimación de la diversidad de especies en la zona. Diferentes variantes se pueden aplicar para adecuar el protocolo tanto a los objetivos de un proyecto como al tipo de ambiente a relevar.

* Se deben escoger los sitios donde se realizarán los muestreos. Esto se puede realizar en forma remota o presencial cuando se realicen muestreos previos.

* En cada sitio se deben escoger los hábitats más adecuados donde colocar las redes. Los hábitats, en orden de calidad y probabilidad de captura, pueden ser cursos o cuerpos de agua (e.g., remansos de arroyos y ríos o lagunas), sendas y caminos en el interior de bosques, bordes de bosques o vegetación, claros de bosque y zonas abiertas como prados y llanuras, entre otros. 
* Se debe muestrear cada sitio al menos durante 3 noches. Se puede aumentar la cantidad de noches según las condiciones atmosféricas reinantes. No se recomienda extender el muestreo por muchas noches en un mismo punto para evitar el acostumbramiento de los individuos a la presencia de las redes. En ese caso, es recomendable cambiar de posición las redes cada noche, aunque esto puede incrementar el esfuerzo de trabajo de campo y reducir los tiempos para otras actividades en el lugar.

* En cada sitio se recomienda desplegar entre 6 y 10 redes repartidas entre los diferentes microambientes. Las redes pueden ser de la misma o diferente longitud, pero deben registrarse las longitudes para poder calcular el esfuerzo de muestreo.

* Las redes se abrirán media hora antes de la caída del sol y se dejarán abiertas durante, al menos, seis horas, correspondientes con el período de mayor actividad diaria de los murciélagos. Realizar muestreos preliminares permitirá obtener información clave para planificar estudios ecológicos. Se recomienda dejar abiertas las redes toda la noche para obtener una mejor idea del nivel diario de actividad y para aumentar el registro de especies raras. La decisión por un tiempo de apertura de redes determinado implica que se continúe con el mismo en los muestreos subsiguientes.

* Las redes se revisarán preferentemente cada 15 ó 30 minutos a fin de remover los individuos capturados. Estos tiempos pueden variar en relación con las tasas de captura observadas, al igual que la cantidad de personas que lo hagan. Si se manejan más de cinco redes será necesario contar con la ayuda de asistentes.

* Los individuos capturados serán removidos cuidadosamente de la red usando guantes de cuero. Cada individuo será colocado en una bolsa de tela blanda que no lo dañe; la bolsa estará limpia y su tamaño será acorde al del ejemplar capturado. Así, el murciélago será trasladado hasta el lugar donde se lo medirá y procesará para la identificación.

* Desde el inicio hasta el final del muestreo se deben mantener las redes libres de ramas, hojas o insectos que puedan hacerla visible. Sobre todo, esto es importante al final para evitar roturas al guardarlas o demoras al reutilizarlas; así también se prolonga la vida útil de las redes.

* Se deben evitar noches de luna llena o estados avanzados de crecientes lunares y noches frías $\left(<10^{\circ} \mathrm{C}\right)$. Si se presentan lluvias intensas, cerrar las redes y abrirlas luego de la lluvia, o continuar el muestreo en la siguiente noche.

* Los resultados se deben presentar destacando los niveles de esfuerzo de muestreo, las tasas de captura, los análisis de eficiencia y los estimadores de riqueza empleados.

\section{CONSIDERACIONES FINALES}

Es recomendable usar métodos estanda_ rizados que puedan ser repetidos de forma confiable en diferentes hábitats, en diversas épocas del año y por distintos investigadores. Utilizar una misma metodología básica permitirá comparar diferentes estudios independientes para ubicarlos dentro de un contexto más amplio. Se espera que los procedimientos aquí descriptos faciliten la estimación de la diversidad de murciélagos y la realización de análisis comparativos de datos que provengan de una misma o de diferentes regiones o que atiendan diferentes temáticas dentro de un marco en común. A la vez, con esta propuesta se espera estimular el estudio de los murciélagos en zonas subtropicales y templadas, apostando a la generación de conocimiento que aporte a la conservación de este grupo tan importante en nuestros ecosistemas.

Agradecimientos. Quiero agradecer enormemente al Dr. Luis F. Aguirre, al Dr. Rubén M. Barquez, al Dr. Mariano Sánchez, a la Dra. M. Cecilia Castilla y a la Dra. M. Ayelén Lutz, quienes aportaron mejoras, consejos y su propia experiencia de años de campo en el manejo de redes de niebla para estudiar estos fascinantes animales, y gracias a quienes este manuscrito mejoró notablemente. Ágradezco el apoyo económico de Idea Wild, Bat Conservation International, Consejo de Investigación de la Universidad Nacional de Salta y al Centro de Investigaciones Básicas y Aplicadas de la Universidad Nacional de Jujuy por brindarme el espacio de trabajo y apoyo. Agradezco al editor principal y asociados por los aportes y observaciones realizadas para mejorar este trabajo. 


\section{REFERENCIAS}

Anthony, E. L. P., and T. H. Kunz. 1977. Feeding strategies of the little brown bat, Myotis lucifugus, in southern New Hampshire. Ecology 58:775-786.

Barquez, R. M., M. A. Mares, and J. K. Brown. 1999. The bats of Argentina. Special Publications. Museum of Texas Tech University. Number 42. Pp. 275.

Barquez, R. M., and M. M. Díaz. 2001. Bats of the argentine Yungas: a systematic and distributional analysis. Acta Zoológica Mexicana 82:29-81.

Barquez, R. M., M. M. Díaz, and R. A. Ojeda (eds.). 2006. Mamíferos de Argentina. Sistemática y Distribución. Sociedad Argentina para el Estudio de los Mamíferos (SAREM), Tucumán, Argentina.

Barquez, R. M., M. S. Sánchez, and J. C. Bracamonte. 2009. Nueva especie de Eptesicus (Chiroptera, Vespertilionidae) para Argentina. Mastozoología Neotropical 17(1):147-152.

Bergallo, H. G., C. E. L. Esbérard, M. A. R. Mello, V. Lins, R. Mangolin, G. G. S. Melo, et al. 2003. Bat Species Richness in Atlantic Forest: What Is the Minimum effort? Biotropica 35:278-288.

Bracamonte, J. C. 2010. Murciélagos de Bosque Montano del Parque Provincial Potrero de Yala, Jujuy, Argentina. Mastozoología Neotropical 17(2):361-366.

Bracamonte, J. C. 2013. Hábitos alimenticios de un ensamble de murciélagos insectívoros aéreos de un bosque montano en las Yungas Argentinas. Chiroptera Neotropical 19(1):1157-1162.

Bracamonte, J. C., and M. A. Lutz. 2013. Nuevos registros de Eumops dabbenei ampliación de la distribución y comentarios sobre su ecología. Mastozoología Neotropical 20(1):1-4.

Carvalho, W. D. de, L. N. Freitas, G. P. Freitas, J. L. Luz., L. de Moraes Costa, et al. 2011. Efeito da chuva na captura de morcegos em uma ilha da costa sul do Rio de Janeiro, Brasil. Chiroptera Neotropical 17:808-816.

Castilla, C. M., R. Torres, and M. M. Díaz. 2013. Murciélagos de la provincia de Córdoba, Argentina: riqueza y distribución. Mastozoología Neotropical 20(2):117-129.

Colwell, R. K, and J. A. Coddington. 1994. Estimating terrestrial biodiversity through extrapolation. Philosophical Transactions of the Royal Society of London. Series B 345:101 -118.

Constantine, D. G. 2009. Bat rabies and other lyssavirus infections. Reston, Va., U.S. Geological Survey Circular 1329. Pp. 68.

Díaz, M. M., and R. M. Barquez. 2009. Primer registro de Micronycteris microtis (Phyllostomidae, Phyllostominae) para la Argentina. Chiroptera Neotropical 15(2):461-465.

Díaz, M. M., D. A. Flores, and R. M. Barquez. 1998. Instrucciones para la preparación y conservación de mamíferos. Programa de Investigaciones de Biodiversidad Argentina, Publicaciones Especiales N 1. Facultad de Ciencias Naturales e Instituto Miguel Lillo, Universidad Nacional de Tucumán y CONICET.

Emmons, L., M. J. Swamer, A. Vargas Espinoza, M. Tschapka, et al. 2006. Las comunidades de murciélagos de bosque y sabana del Parque Nacional Noel Kempff. Revista Boliviana de Ecología 19:47-57.

Esbérard, C. E. L. 2009. Capture sequence and relative abundance of bats during surveys. Zoología 26:103-108.

Esbérard, C. E. L., and H. G. Bergallo. 2005. Coletar morcegos por seis ou doze horas a cada noite? Revista Brasileira de Zoologia 22:1095-1098.

Estrada, A., and R. Coates-Estrada. 2002. Bats in continuous forest, forest fragments and in an agricultural mosaic habitat-island at Los Tuxtlas, Mexico. Biological Conservation 103:237-245.

Fenton, M. B., Acharya, L., Audet, D., Hickey, M. B. C., Merriman, C., Obrist, M. K., and D. M. Syme. 1992. Phyllostomid bats (Chiroptera: Phyllostomidae) as indicators of habitat disruption in the Neotropics. Biotropica 24:440-446.

Finnemore, M., and P. W. Richardson. 2004. Catching bats. Pp. 41-48 en Mitchell-Jones, A. J. y A. P. Mcleish (eds.). Bat Workers' Manual. 3rd Edition, Joint Nature Conservation Committee, Peterborough.

Fleming, T. H., C. Geiselman, and W. J. Kress. 2009. The evolution of bat pollination: a phylogenetic perspective. Annals of Botany 104(6):1017-1043

Frencke, B. Von, and R. M. R. Barclay. 1987. Bat activity over calm and turbulent water. Canadian Journal of Zoology 65:219-222.

Galarza, M. I., and L. F. Aguirre (eds.). 2006. Métodos estandarizados para el estudio de murciélagos en bosques montanos. Centro de Estudios en Biología Teórica y Aplicada. Cochabamba, Bolivia. Pp. 63.

Gannon, M. R., and M. R. Willig. 1997. The effect of lunar illumination on movement and activity of the red fig-eating bat (Stenoderma rufum). Biotropica 29:525-529.

Jantzen, M. K., and M. B. Fenton. 2013. The depth of edge influence among insectivorous bats at forest - field interfaces. Canadian Journal of Zoology 91:287-292.

Jones, G., D. Jacobs, T. H. Kunz, M. R., Willig, and P. A. Racey. 2009. Carpe noctem: the importance of bats as bioindicators. Endangered Species Research 8:93-115.

Jouard, S. 2007. Effect of bat exclusion on patterns of seed rain in tropical rain forest in French Guiana. Biotropica 39: 510-518.

Kingston, T. 2009. Analysis of species diversity of bat assemblages. Pp. 195-215 en Kunz, T. H. y S. Parson (eds.). Ecological and behavioral methods for the study of bats. The John Hopkins University Press. Baltimore.

Klingbeil, B. T., and M. R. Willig. 2010. Seasonal differences in population-, ensemble- and community-level responses of bats to landscape structure in Amazonia. Oikos 119:1654-1664. 
Kunz, T. H. y A. Kurta. 1990. Capture methods and holding devices. Pp. 1-30 en Kunz, T. H. (ed.). Ecological and behavioral methods for the study of bats. Smithsonian Institution Press. Washington D.C. London.

Kunz, T. H., R. Hodkison, and C. D. Weise. 2009. Methods of capturing and handling bats. Pp. 3-35 en Kunz, T. H. y S. Parson (eds.). Ecological and behavioral methods for the study of bats. The John Hopkins University Press. Baltimore.

Kunz, T. H, E. Braun De Torrez, D. Bauer, T. Lovoba, and T. H. Fleming. 2011. Ecosystem services provided by bats. Annals of the New York Academy of Sciences 1223:1-38.

Lang, A. B., E. K. V. Kalko, H. Römer, C. Bockholdt, and D. K. N. Dechmann. 2006. Activity levels of bats and katydids in relation to the lunar cycle. Oecologia 146:659-66.

LaVal, R. K., and H. S. Fitch. 1977. Structure, movements and reproduction in three Costa Rican bat communities. Occasional Papers of the Museum of Natural History. University of Kansas. Lawrence. 69:1-27

Lisón, F., and J. F. Calvo. 2011. The significance of water infrastructures for the conservation of bats in a semiarid Mediterranean landscape. Animal Conservation 14:533-541.

Longino, J. T., J. A. Coddington, and R. K. Colwell. 2002. The ant fauna of a tropical rain forest: estimating species richness three different ways. Ecology 83:689-702.

López Berrizbeitia, M. F., and M. M. Díaz. 2013. Diversidad de murciélagos (Mammalia, Chiroptera) en la ciudad de Lules, Tucumán. Acta Zoológica Mexicana (N.s.) 29(1):234-239.

McNab, B. K. 1982. Evolutionary alternatives in the physiological ecology of bats. Pp. 151-200 en Kunz, T. H. (ed.). Ecology of Bats. New York: Plenum Press.

Magurran, A. E. 2004. Measuring biological diversity. Blackwell Publishing, London.

Medellín, R. 1993. Estructura y diversidad de una comunidad de murciélagos en el trópico húmedo mexicano. Pp. 333350 en Medellín, R. y G. Ceballos (eds.). Avances en el estudio de los mamíferos de México. Publicaciones especiales. Asociación Mexicana de Mastozoología, A. C. México, Distrito Federal. Medellín.

Medellín, R. A., M. Equihua, and M. A. Amín. 2000. Bat diversity and abundance as indicators of disturbance in Neotropical rainforest. Conservation Biology 14(6):1666-1675.

Mello, M. A. R., E. K. V Kalko, and W. R. Silva. 2008. Diet and abundance of the bat Sturnira lilium (Chiroptera) in a Brazilian Montane Atlantic Forest. Journal of Mammalogy 89:485-492.

Moreno, C. E. 2001. Métodos para medir la biodiversidad. MyT, Manuales y Tesis SEA. Zaragoza. España.

Moreno, C. E., and G. Halffter. 2000. Assessing the completeness of bat biodiversity inventories using species accumulation curves. Journal of Applied Ecology 37:149-158.

Morrison, D. W. 1978. Lunar phobia in a Neotropical fruit bat Artibeus jamaicensis (Chiroptera, Phyllostomatidae). Animal Behavior 26:853-855

Palmeirim, J. M., and K. Etheridge. 1985. The influence of man-made trails on foraging by tropical frugivorous bats. Biotropica 17:82-83.

Patterson, B. D., M. R. Willig, and R. D. Stevens. 2003. Trophic strategies, niche partitioning, and patterns of ecological organization. Pp. 536-579 en Kunz, T. H. and M. B. Fenton (eds.). Bat Ecology. University of Chicago. Press, Illiniois.

Peters, S. L., J. R. Malcolm, and B. L. Zimmerman. 2006. Effects of selective logging on bat communities in the Southeastern Amazon. Conservation Biology 20(5):1410-1421.

Rinehart, J. B., and T. H. Kunz. 2001. Preparation and deployment of canopy mist nets made by Avinet. Bat Research News 42(3):85-88.

Rydell, J. 1993. Variation in foraging activity of an aerial insectivorous bat during reproduction. Journal of Mammalogy 74:503-509.

Saldaña-Vázquez, R. A., and M. A. Munguía-Rosas. 2013. Lunar phobia in bats and its ecological correlates: A metaanalysis. Mammalian Biology - Zeitschrift für Säugetierkunde 78:216-219.

Sánchez, M. S., and N. P. Giannini. 2013. Altitudinal patterns in two syntopic species of Sturnira (Mammalia: Chiroptera: Phyllostomidae) in the montane rain forests of Argentina. Biotropica 46:1-5.

Sánchez, M. S., L. V. Carrizo, N. P. Giannini, and R. M. Barquez. 2012a. Seasonal patterns in the diet of frugivorous bats in the subtropical rainforests of Argentina. Mammalia 76(3):269-275. doi: 10.1515/mammalia-2011-0059.

Sánchez, M. S., N. P. Giannini, and R. M. Barquez. 2012b. Bat frugivory in two subtropical rain forests of Northern Argentina: Testing hypotheses of fruit selection in the Neotropics. Mammalian Biology - Zeitschrift Für Säugetierkunde 77(1):22-31.

Sandoval, M. L., C. A. Szumik, and R. M. Barquez. 2010. Bats and marsupials as indicators of endemism in the Yungas forest of Argentina. Zoological Research 31(6):633-644.

Sandoval, M. L., and R. M. Barquez. 2013. The Chacoan bat fauna identity: Patterns of distributional congruence and conservation implications. Revista Chilena de Historia Natural 86:75-94.

Schipper, J., J. S. Chanson, F. Chiozza, et al. 2008. The status of the world's land and marine mammals: diversity, threat, and knowledge. Science 322:225-230.

Sikes, R. S., W. L. Gannon, and The Animal Care and Use Committee of the American Society of Mammalogists. 2011. Guidelines of the American Society of Mammalogists for the use of wild mammals in research. Journal of Mammalogy 92(1):235-253.

Simmons, N. B., and R. S. Voss. 1998. The mammals of Paracou, French Guyana: A Neotropical lowland rain forest 
fauna. Part I: Bats. Bulletin of the American Museum of Natural History 237:219.

Simmons, N. B., and R. S. Voss. 2009. Collection, preparation, and fixation of bats specimens and tissues. Pp 849-867 en Kunz, T. H. y S. Parson (eds.). Ecological and behavioral methods for the study of bats. The John Hopkins University Press. Baltimore.

Swift, S. M. 1980. Activity patterns of Pipistrelle bats (Pipistrellus pipistrellus) in north-east Scotland. Journal of Zoology of London 190:285-295.

Thomas, D. W., and Laval, R. K. 1988. Census and survey techniques. Pp. 77-87 en Kunz, T. H. (ed.). Ecological and behavioral methods for the study of bats. Smithsonian Institution Press, Washington, D.C.

Tschapka, T. M. 1998. A compact and flexible method for mist-netting bats in the subcanopy and canopy. Bat Research News 39:140-141.

Voigt, C. C., K. Schneeberger, S. L. Voigt Heucke, and D. Lewanzik. 2011. Rain increases the energy cost of bat flight. Biology Letters 7:793-795. 